\title{
Estimating Available Bandwidth in Cooperative Multi-hop Wireless Networks
}

\author{
Wei Feng, Jian Liu, Jiannong Cao, Liang Yang, and Qin Xin \\ China Transportation Telecommunication and Information Center, China \\ Hong Kong Polytechnic University, Hong Kong \\ University of the Faroe Islands, Denmark \\ \{fengwei, liujian\}@ettic.cn, \{csjcao,csliangy\}@comp.polyu.edu.hk, \\ qinxasetur.fo
}

\begin{abstract}
Estimating available bandwidth (EAB) is an important and challenging task for providing QoS support in wireless networks. Existing works on EAB did not consider cooperative communication which can improve available bandwidth of wireless networks. To fill this void, this paper studies the problem of estimating available bandwidth in cooperative multi-hop wireless networks, which is formulated as an optimization problem, called Cooperative Available Bandwidth Estimation problem (Coop-ABE). Given a new connection request, the Coop-ABE problem targets at finding the maximum available bandwidth that can be provided to the new connection subject to that existing flows are kept free from interference. We propose a centralized algorithm to solve the Coop-ABE problem.
\end{abstract}

Keywords: Cooperative, QoS, routing, scheduling.

\section{Introduction}

Estimating available bandwidth (EAB) is an important and challenging task for providing QoS support in multi-hop wireless networks. For example, before admitting a new connection request to a multi-hop wireless network, it is important for us to know the maximum available bandwidth that the network can provide to this new connection. If the maximum available bandwidth is larger than the bandwidth demand of the new connection, the new connection will be accepted. Otherwise, it will be blocked. Moreover, cooperative communication is a new technology, which can improve poor links' transmission capacity with other nodes' help. Hereafter, we denote the node which helps other links retransmit packets by helper node. The cooperative communication provides us a new method to improve the available bandwidth of a multi-hop wireless network, but no existing works [1],[2],[3] on EAB consider it. This paper tries to fill this void, and studies how to estimate available bandwidth in cooperative multi-hop wireless networks.

We formulate the problem of estimating available bandwidth in cooperative multihop wireless networks as an optimization problem, called Cooperative Available Bandwidth Estimation problem (Coop-ABE). Compared with existing works about 
EAB problem, the Coop-ABE problem needs to address following new issues. For example existing works only consider routing and scheduling issues, and they do not consider how to select helper nodes for forwarding links in the routing path. However, in the Coop-ABE problem, we need to consider how to jointly select routing path and helper nodes to achieve the maximum available bandwidth. Moreover, the Coop-ABE problem needs to consider more complicated interference relationship and capacity model.

We make following contributions while solving the Coop-ABE problem. First, we construct a new virtual graph to represent the new interference relationship and new capacity model. In the new virtual graph, we add some virtual links to represent the links working in the cooperative transmission mode. We redefine the interference relationship and capacity for these virtual links. By doing this, we significantly simplify the interference relationship among links and the capacity model. Second, we transform the Coop-ABE problem to a simpler problem which targets at finding a single-path rouging and the corresponding scheduling scheme in the virtual graph. Third, we propose a centralized polynomial approximation algorithm to solve the Coop-ABE problem, referred to as the centralized Coop-ABE algorithm.

\section{System Model and Problem Formulation}

\subsection{System Model}

In this paper, we consider a cooperative multi-hop wireless network. We adopt the $\Omega(1)$ model introduced in [4] to bound the distance between node pairs: the distance between any two nodes may not fall below a constant $d_{0}$. This assumption is reasonable because in the real wireless network there are physical limitations on how close to each other two nodes can be placed. We model the network as an undirected graph $G(V, E)$, where $V$ denotes a set of $n$ nodes, and $E$ denotes a set of $m$ links.

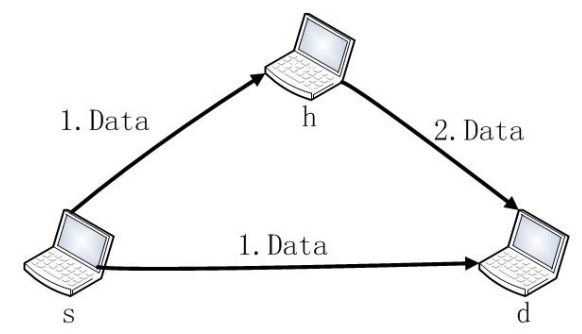

Fig. 1. DF cooperation scheme

We assume that a Decode-and-Forward cooperation scheme (DF) is adopted to improve poor links' transmission capacity. The capacity for links working on DF mode under the two-time-slot structure is given [5].

$$
C_{D F}(s, h, d)=W * I_{D F}(s, h, d)
$$




$$
I_{D F}(s, h, d)=\frac{1}{2} * \min \left\{\log _{2}\left(1+\operatorname{SINR}_{s h}\right), \log _{2}\left(1+\operatorname{SINR}_{s d}+\operatorname{SINR}_{h d}\right)\right\}
$$

The capacity for direct transmission is described as follows.

$$
C_{\text {direct }}(s, d)=W * \log _{2}\left(1+\operatorname{SINR}_{s d}\right)
$$

To simplify our analysis, we use the Additive White Gaussian Noise (AWGN) model in [6] to approximate one link's SINR.

$$
\operatorname{SINR}_{i j}=\frac{P}{d\left(l_{i j}\right)^{\alpha} N_{0} W}
$$

Here, $W$ is the bandwidth, $N_{0}$ is white noise level, $\alpha$ is path loss factor, $d\left(l_{i j}\right)$ is the distance between node $i$ and $j$. Because $W, N_{0}$, and $\alpha$ are all constants, each link's SINR and capacity are fixed.

\subsection{Problem Formulation}

We formally define Coop-ABE problem as follows.

Definition1. (Cooperative Available Bandwidth Estimation problem, Coop-ABE) Given a network $G(V, E)$, each link's $S I N R$ and $A T F$, a set of existing flows, and a new connection request $\rho(s, t)$ with bandwidth requirement $R B W$, the problem is to determine whether request $\rho(s, t)$ can be accommodated by finding the maximum available bandwidth that the network can provide to connection $\rho(s, t)$ subject to that existing flows are kept free from interfere. We need to find a cooperative routing path $P$ from $s$ to $t$, and a scheduling scheme for links in $P$ to achieve the maximum available bandwidth. We mathematically describe the problem as follows.

Objective: $\operatorname{Maximize}(B W(P))$

subject to:

- Path bandwidth constraint:

$$
B W(P)=C\left(l_{i, i+1}, h_{i}\right) * T F\left(l_{i, i+1}, h_{i}, \rho\right)
$$

- Flow conservation constraint:

$$
\forall l_{i, i+1}, l_{j, j+1} \in P, C\left(l_{i, i+1}, h_{i}\right) * T F\left(l_{i, i+1}, h_{i}, \rho\right)=C\left(l_{j, j+1}, h_{j}\right) * T F\left(l_{j, j+1}, h_{j}, \rho\right)(6)
$$


- Feasible scheduling constraint:

$$
\forall l \in I S(P), \sum_{e \in I S(l)} T F(e, \rho) \leq A T F(l)
$$

- Relay constraint:

$$
\forall i, j, m \in V, \$ \sum_{m \in V} x(m, P)+\sum_{l_{i j} \in P} y\left(m, l_{i j}, P\right) \leq 1
$$

Here, $B W(P)$ denotes the available bandwidth of path $P, I S(P)$ denotes the set of links interfering with links in $P, I S(P)=\cup_{l \in P} I S(l) . C\left(l_{i, i+1}, h_{i}\right)$ denotes the capacity of link $l_{i, i+1}$ working in the cooperative transmission mode with node $h_{i}$ as its helper. $T F\left(l_{i, i+1}, h_{i}, \rho\right)$ denotes the time fraction consumed by link $l_{i, i+1}$ and its helper node $\mathrm{h}_{i}$ when it works in the cooperative transmission mode with node $\mathrm{h}_{i}$ as its helper. The first constraint defines the path available bandwidth. The second constraint denotes the flow conservation constraint. The third constraint denotes each node within interference range of links in $\$ \mathrm{P} \$$ should not be interfered. The fourth constraint denotes that any node can act only once either as a forwarder or a helper in $P$.

\section{$3 \quad$ Algorithm Design and Analysis}

\subsection{Construct Virtual Graph}

In this subsection, we transform the Coop-ABE problem to a simpler problem by constructing a virtual graph. We show the transformation by an example in figure2, in which node 2 is a helper for link $1_{13}$ and $1_{56}$. We construct a virtual graph $G^{\prime}\left(V^{\prime}, E^{\prime}\right)$ shown in the right graph of figure 2 by following steps.

1) Add virtual nodes and virtual links: We add virtual nodes and virtual links to denote the potential helpers and links working in the cooperative transmission mode. For example, in figure2, node 2 is a potential helper for link $1_{56}$, we add a virtual node 8 to represent the helper node 2 , and two virtual links $1_{58}$ and $1_{86}$ to denote links between node 5 and node 6 working in the cooperative transmission mode. We also add virtual node 7 and virtual links $l_{17}$ and $l_{73}$ since node 2 also works as helper node for links $l_{13}$. To simplify our presentation, we define the set of virtual links in $G^{\prime}$ as Virtual link Set of $G^{\prime}$, denoted by $\operatorname{VS}\left(G^{\prime}\right)$. We define the set of original links in $G^{\prime}$ as Original link Set, denoted by $O S\left(G^{\prime}\right)$. Given a virtual link v, we also define a link set, L(v), to denote the original links that virtual link $\mathrm{v}$ represents. For example, in figure2, $\mathrm{L}\left(1_{17}\right)=\left\{1_{12}, 1_{13}\right\}$. 
2) Label virtual links: We label virtual links with three metrics, including capacity, ATF and hop-count. Because we divide the transmission of one original link 1 into two virtual links, the transmission capacity of a virtual link is defined as twice as that of link 1 working in the cooperative transmission mode. For example, in figure2, we define the transmission capacity of virtual link $l_{17}$ as $C\left(1_{17}\right)=C\left(1_{73}\right)=2 * C_{D F}(1,2,3)$. When link $\$ 1 \$$ works in the cooperative transmission mode, it needs to work cooperatively with other two links. So we set ATF of a virtual link $v$ as the minimum ATF of the original links that link v represents. For example, in figure2, we set ATF of virtual link $l_{17}$ as $A T F\left(l_{17}\right)=\min \left\{A T F\left(l_{12}\right), A T F\left(l_{13}\right)\right\}$. Because the length of a cooperative routing path is equal to that of a routing path $\mathrm{P}$, we set hop-count for original link as $\$ 1 \$$ and that for virtual link as 0.5 .

3) Redefine interference relationship: We redefine the interference relationship in the virtual graph by redefining original and virtual links' interference link set (IS). We define IS of an original link 1 as that of original link 1 and virtual links which include original links interfering with link 1. For example, in figure2, in original graph, we suppose that $\operatorname{IS}\left(1_{13}\right)=\left\{1_{12}, 1_{23}, 1_{34}\right\}$. In virtual graph, we set $\operatorname{IS}\left(1_{13}\right)=\left\{1_{12}, 1_{23}, 1_{34}, 1_{17}, 1_{73}\right\}$. The IS of a virtual link $\mathrm{v}$ is defined as set of links interfering with original links included in $\mathrm{v}, I S(v)=\cup_{l \in L(v)} I S(l), \mathrm{L}(\mathrm{v})$ denotes original links included in v. For example, in figure2, $\operatorname{IS}\left(1_{17}\right)=\left\{1_{12}, 1_{23}, 1_{34}, 1_{13}, 1_{73}\right\}$.

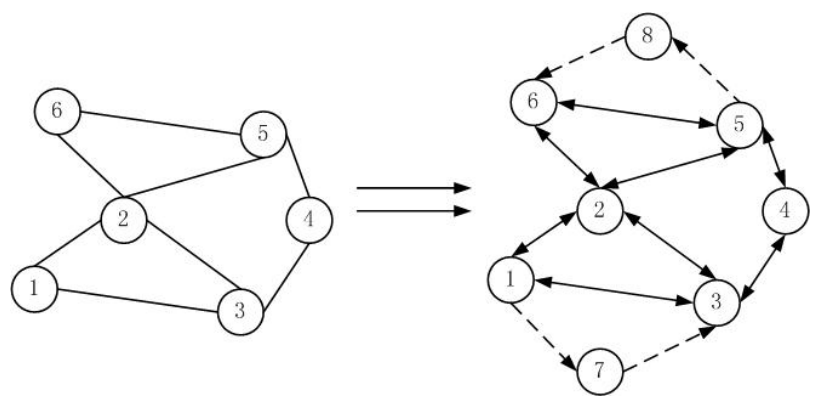

Fig. 2. Example of constructing virtual graph

Based on concept of virtual graph, we transform Coop-ABE problem to a simpler routing problem, which seeks a single-path routing $P$ and a scheduling scheme $\$ S \$$ to achieve the maximum available bandwidth in the virtual graph.

\subsection{Centralized Coop-ABE Algorithm}

We propose a centralized algorithm, called centralized Coop-ABE algorithm to solve the Coop-ABE problem. To guarantee that transmission on link 1 does not interfere with other links, link 1 cannot occupy time fraction more than ATF of links within its interference range. We define one metric, called Bottle-neck Available Time Fraction 
(BTF) to denote the available time fraction of link $\$ 1 \$$ while considering interference constraint, $B T F(l)=\min _{e \in I S(l)} A T F(e)$. We define another metric, called Bottleneck Bandwidth (BBW) to denote the available bandwidth of link 1 when considering the interference constraint, $B B W(l)=\min _{e \in I S(l)}\{C(e) * B T F(e)\}$. Intuitively, we should select links with high BBW to achieve high available bandwidth. We construct a sub-graph, called reserved graph to select links with high BBW. We divide the centralized Coop-ABE algorithm into three phases as follows.

1) Construct reserved graph: We first construct a virtual graph $G^{\prime}\left(V^{\prime}, E^{\prime}\right)$ for the network $\mathrm{G}(\mathrm{V}, \mathrm{E})$ according to rules in above subsection. To solve the duplicated relay node selection problem, we combine original node and its corresponding helper nodes as a supper node to represent that these links share a helper node. For example, nodes $2,7,8$ in the virtual graph of figure 2 are combined as a super node 2 in the left graph in figure5. Because we need to select links with high \$BBW\$, we construct a sub-graph, called reserved graph, $G^{*}\left(V^{*}, E^{*}, m i d\right)$ in the right graph of figure3, which only includes the links with BBW higher than the threshold mid. We use binary search method to update the threshold value \$mid\$ until the shortest cooperative routing $\mathrm{P}$ from node $\mathrm{s}$ to $\mathrm{t}$ in the reserved graph $G^{*}$ is found. We formally define reserved graph as follows.

Definition 2. (Reserved Graph) Given a weighted graph $G(V, E)$, and a threshold mid, Reserved Graph $G^{*}\left(V^{*}, E^{*}\right.$, mid $)$ is defined as a sub-graph of $\mathrm{G}$, which contains links with weights no less than mid, $w(e) \geq$ mid .

Algorithm 1. Centralized Coop-ABE

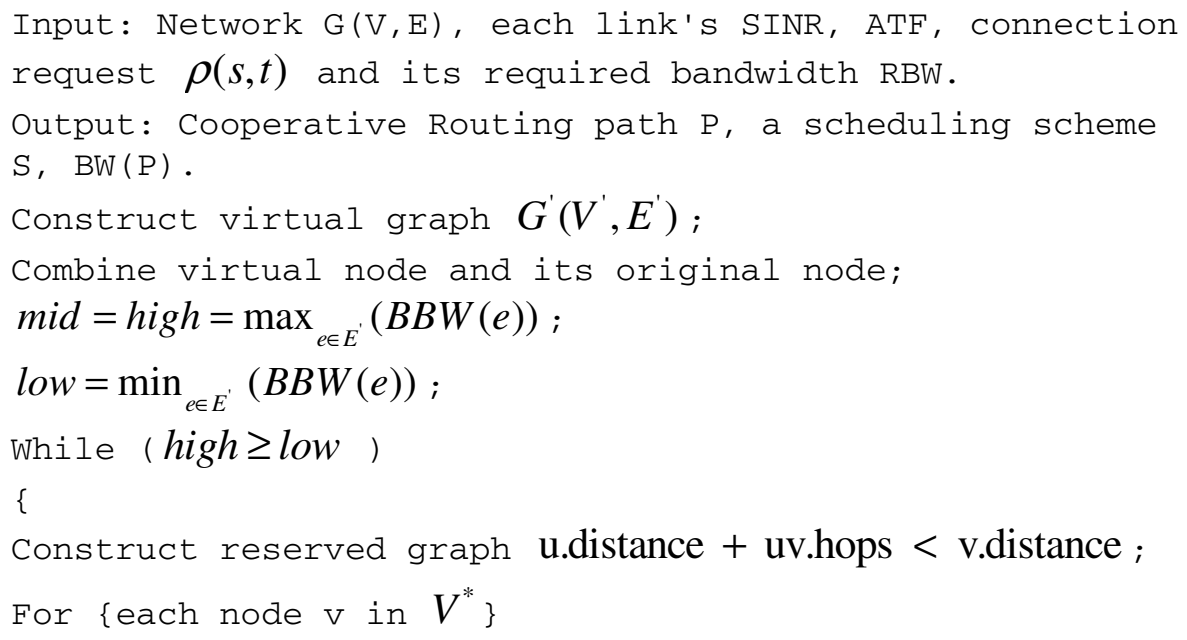



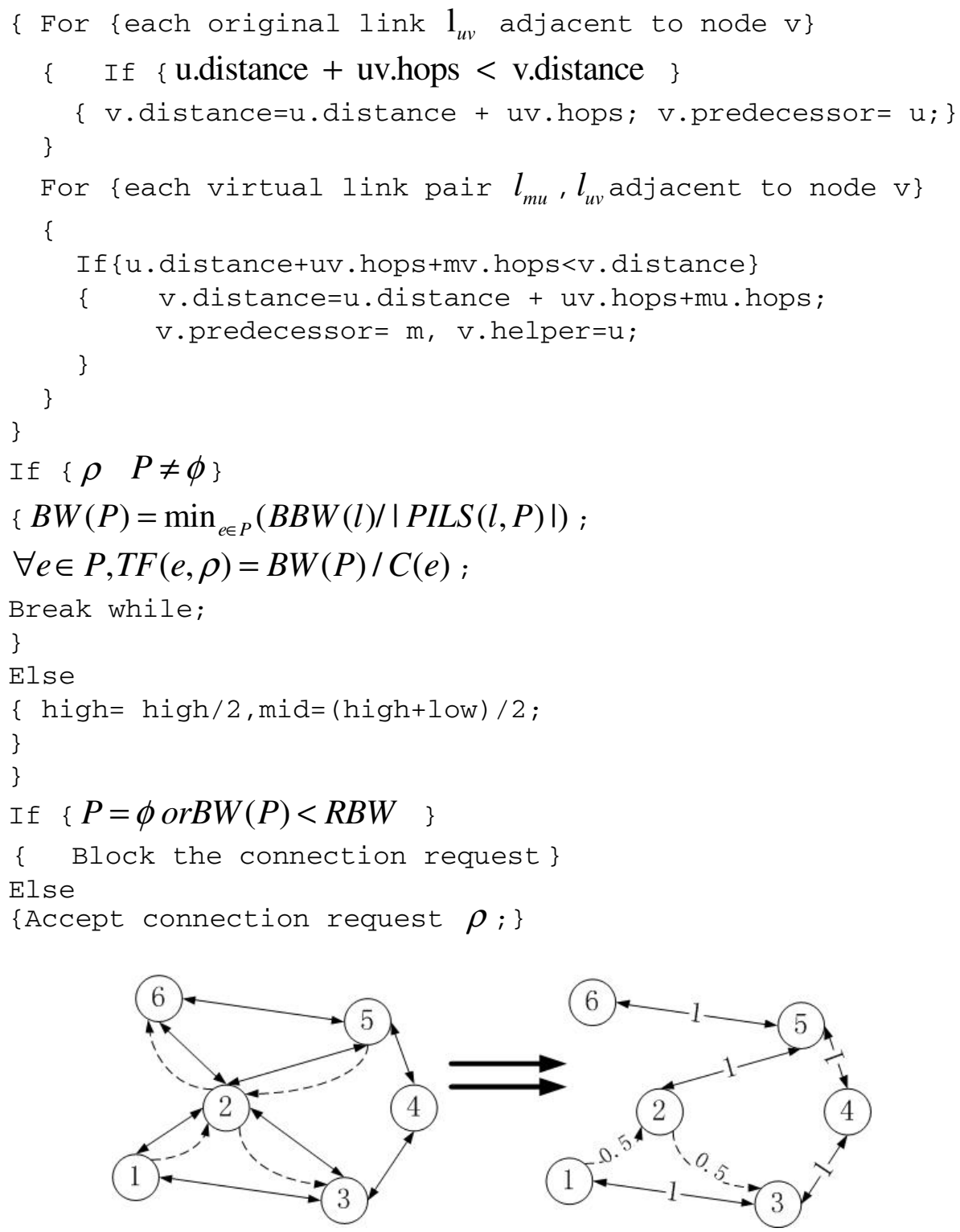

Fig. 3. Example of reserved graph

2) Find the shortest path: We also prefer path with short length because the shortest path leads to less opportunity of interference. We modify Bellman Ford algorithm to find the shortest path in reserved graph $G^{*}\left(V^{*}, E^{*}, B\right)$.

3) Estimate available bandwidth \} \} In this phase, we estimate available bandwidth of the selected cooperative routing path P. We define a link set, called Path Interference Link Set (PILS) to denote the links interfering with link 1 in path P. 
Definition 3. (Path Interference Link Set, PILS) Given a link 1 and a routing path $\mathrm{P}$, PILS is defined as a set of links in $\mathrm{P}$ interfering with link $1, \$$ $P I L S(l, P)=\{e \in E \mid e \in P, e \in I S(l)\}$.

When considering interference from neighboring links in $\mathrm{P}$, link 1 in path $\mathrm{P}$ can at most achieve following bandwidth, BW $(1)=\mathrm{BBW}(1) /|\operatorname{PILS}(1, \mathrm{P})|$. The available bandwidth of path $\mathrm{P}$ is determined by the bottle-neck link, $\min _{e \in P}(B B W(e) / P I L S(e, P))$. We check whether the connection's bandwidth requirement is satisfied or not. If it is satisfied, we accept the new connection. Otherwise, the new connection is blocked.

\section{Conclusion}

In this paper, we study the problem of estimating available bandwidth in coop- erative multi-hop wireless networks. We propose centralized algorithms to solve the Coop$\mathrm{ABE}$ problem. We prove that the centralized algorithm can achieve a constant approximation ratio.

\section{References}

1. Zhai, H., Fang, Y.: Impact of routing metrics on path capacity in multi-rate and multi-hop wireless ad hoc networks. In: Proceedings of the 14th IEEE International Conference on Network Protocols (ICNP 2006), Santa Barbara, USA (2006)

2. Zhu, C., Corson, M.: Qos routing for mobile ad hoc networks. In: Proceedings of IEEE INFOCOM 2002, New York, USA (2002)

3. Chen, F., Zhai, H., Fang, Y.: Available bandwidth in multirate and multihop wireless ad hoc networks. IEEE Journal on Selected Areas in Communications 28, 299-307 (2010)

4. Kuhn, F., Wattenhofer, R., Zollinger, A.: Worstcase optimal and average case efficient geometric adhoc routing. In: Proceedings of ACM MobiHoc 2003, Annapolis, Maryland, USA (2003)

5. Laneman, J.N., Tse, D.N.C., Wornell, G.W.: Cooperative diversity in wireless networks: Efficient protocols and outage behavior. IEEE Transactions on Information Theory 50, 3062-3080 (2004)

6. Chafekar, D., Kumar, V.S.A., Marathe, M.V., Parthasarathy, S., Srinivasan, A.: Approximation algorithms for computing capacity of wireless networks with sinr constraints. In: Proceedings of IEEE INFOCOM 2008, Phoenix, USA (2008) 